\title{
Role of MRCP in Assessment of Biliary Anatomy in Potential Living Liver Transplant Donors
}

\author{
Medhat M. Reffat ${ }^{\mathrm{a}}$, Ahmed E. Shalan ${ }^{\mathrm{a}}$, Shehab El-Din Shibl ${ }^{\mathrm{b}}$ \\ a Department of Radiology, \\ Benha faculty of medicine, \\ Benha University, Egypt. \\ b Department of Radiology, \\ Ahmed Maher Teaching \\ Hospital, Cairo, Egypt \\ Correspondence to: Shehab \\ El-Din Shibl, Ahmed Maher \\ Teaching Hospital, Cairo, Egypt \\ Email: \\ ibrahim.shibl@yahoo.com \\ Received: 23 July 2020 \\ Accepted: 25 March 2021

\begin{abstract}
:
Aim: to assess biliary anatomical variants in potential living liver transplant donors using Yoshida classification. Patients and methods: the study was carried out on 54 potential living liver transplantation donors. The donors were randomly selected and evaluated with preoperative MRCP. All candidates' pattern of biliary tree was classified according to Yoshida classification. Results: The most common biliary tree arborizing pattern was RL type (bifurcation of the right and left ducts), it was seen in 40 candidates $(74.04 \%)$. The second most common pattern was A-PL where right posterior intrahepatic duct joins the left hepatic duct, then they both join the right hepatic duct to form the common hepatic duct, this pattern was seen in 9 candidates (16.66\%). Least common was the trifurcation pattern (APL), which was seen in 5 candidates $(9.25 \%)$. Conclusion: Preoperative MRCP is an essential step in evaluating potential living liver transplant donors.
\end{abstract}

As a non-invasive technique, MRCP can safely and accurately delineate the branching pattern of biliary tree, guiding the surgical team and reducing post-operative complications.

Key words: MRCP, Biliary anatomical variants, Living liver transplant Donors.

\section{List of Abbreviations:}

MRCP: Magnetic Resonance Cholangio-Pancreatography. 


\section{Introduction}

Conventional MRCP is a standard, noninvasive method for evaluating the biliary tree, assessing the extrahepatic bile ducts and dilated intrahepatic bile ducts ${ }^{[1]}$.

In living donor liver transplantation (LDLT), biliary complications appear to be the leading cause of postoperative complications after living donor liver transplantation. ${ }^{[2]}$. Thus, accurate knowledge of the anatomy of intrahepatic ducts is critical to reduce these complications ${ }^{[3]}$.

A comprehensive MR imaging examination is the preoperative imaging modality of choice for living liver donor candidates for accurate definition of intrahepatic biliary anatomy ${ }^{[4]}$. MRCP is essential for all pretransplantation evaluation with high accuracy rate [5] and may guide intraoperative management of the biliary $\operatorname{tract}^{[6]}$.

\section{Patients and Methods}

Type of the study: Observational crosssectional study.

\section{Patients:}

From July 2018 to July 2019, the study was carried out on 54 potential living liver transplantation donors who were enrolled from Benha University hospitals. The donors were randomly selected and evaluated with preoperative MRCP. Biliary anatomical variants were classified according to Yoshida classification. Total number of candidates was 55; 35 candidates were males and 20 candidates were females. Candidates' age ranged between 20-46 years. Mean age was 31 years old $(\mathrm{SD}=$ $6.21)$.

\section{MRCP technique:}

MRCP was performed using a 1.5-Tesla machine. A respiratory-triggered T2W TSE sequence with PACE technique (TR/TE, 1,600/678; flip angle, 170 ; FOV, 400 mm; matrix size, $384 \times 384$; section thickness, $1.6 \mathrm{~mm}$ ) in axial and coronal planes was performed. Data collected was processed with MIP and SSD algorithms. 3D reformatted images were obtained.

\section{Data analysis:}

Anatomical data was classified according to Yoshida Classification ${ }^{[7]}$ for biliary variants.

\section{Ethical Considerations:}

A written consent was taken from all participants in this research and ethical committee approval was obtained. 


\section{Results}

Statistical analysis of results: Sample size was calculated using "EpiInfo" software program. Data, results and statistical analysis were tabulated and calculated using Excel Spreadsheet.

\section{Biliary anatomical variants}

All candidates' pattern of biliary tree was classified according to Yoshida classification.
In our study the results came as follows; the most common biliary tree arborizing pattern was bifurcation of the right and left ducts (RL) with 40 candidates $(74.04 \%)$. The second most common pattern was A-PL where right posterior intrahepatic duct joins the left hepatic duct, then they both join the right hepatic duct to form the common hepatic duct, this pattern was seen in 9 candidates $(16.66 \%)$. Least common was the trifurcation pattern (APL), seen in 5 candidates $(9.25 \%)$ (Table 1$)$.

Table (1): Intrahepatic biliary anatomical variants according to Yoshida classification.

\begin{tabular}{llll}
\hline & Branching variant & Number & Percentage \\
\cline { 2 - 4 } & RL & 40 & $74.04 \%$ \\
A-PL & 9 & $16.66 \%$ \\
APL & 5 & $9.25 \%$ \\
& Total & 54 & $100 \%$ \\
\hline
\end{tabular}

\section{Associations}

During our study, many side findings were discovered incidentally among candidates. These findings are asymptomatic and required no further investigations or management. The following table (2) summarizes these findings and their percentages among candidates.

\section{Biliary tree associations}

The most common biliary tree association was low insertion of cystic duct which was found in about $22 \%$ of candidates. Other findings such as an accessory right hepatic duct draining into either right or left intrahepatic ducts were also encountered. The next table (2) summarizes these findings. 
Table (2): biliary tree anatomical variant associations.

\begin{tabular}{lll}
\hline Association & Number & Percentage \\
\hline Low cystic duct insertion & 12 & $22.2 \%$ \\
Accessory right hepatic duct draining into right intrahepatic duct & 4 & $7.4 \%$ \\
Accessory right hepatic duct draining into left intrahepatic duct & 2 & $3.7 \%$ \\
Aberrant insertion of cystic duct into right hepatic duct & 1 & $1.8 \%$ \\
\hline
\end{tabular}

\section{Hepatic associations}

Hemangiomas were found to be the most common incidental finding among candidates with about $7.4 \%$ prevalence. Simple liver cyst was found in one case (Table 3 ).
Other associations;

Biliary hamartoma, pancreatic divisum and a left adrenal mass were found once for each of them.

Table (3): Summary of associations.

\begin{tabular}{lll}
\hline Association & Number & Percentage \\
\hline Low cystic duct insertion & 12 & $22.2 \%$ \\
Accessory right hepatic duct draining into right intrahepatic duct & 4 & $7.4 \%$ \\
Accessory right hepatic duct draining into left intrahepatic duct & 2 & $3.7 \%$ \\
Liver hemangioma & 4 & $7.4 \%$ \\
Liver cyst & 1 & $1.8 \%$ \\
Biliary hamartoma & 1 & $1.8 \%$ \\
Pancreatic divisum & 1 & $1.8 \%$ \\
Left adrenal mass & 1 & $1.8 \%$ \\
Aberrant insertion of cystic duct into right hepatic duct & 1 & $1.8 \%$ \\
\hline
\end{tabular}

\section{Discussion}

In our study, we used Yoshida classification to interpret our data. Yoshida classification has the advantage of being simple, easily understood and that it uses letter as an abbreviation to anatomical landmarks of the biliary tree. This makes Yoshida classification an easy option for radiologists aiming to detect biliary anatomical variants, and for surgeons in their preoperative and intraoperative management of LDLT cases 
or other similar procedures based on the same anatomical considerations.

In our study; 40 candidates $(74.04 \%)$ showed the commonest RL type, 9 candidates $(16.66 \%)$ showed A-PL type, the trifurcation pattern (APL) came last in 5 candidates $(9.25 \%)$

Other biliary tree variations were found among candidates. Low cystic duct insertion was the most encountered association, seen in 12 candidates $(22.2 \%)$. Accessory right hepatic duct draining into right intrahepatic duct was seen in 4 candidates $(7.4 \%)$. An accessory right hepatic duct draining into left intrahepatic duct was seen in 2 cases (3.7\%). Aberrant insertion of cystic duct into right hepatic duct was seen in 1 candidate $(1.8 \%)$.

A 2013 study reported that there are many normal variants of the biliary anatomy which represent a challenging point in the surgical planning of living donation. Therefore, the donor's biliary system should be analyzed preoperatively to establish bile duct variations, plan the appropriate surgical resection technique and the method for biliary anastomosis. There are many techniques that can be used for pre-operative biliary mapping such as Magnetic
Resonance Cholangiopancreatography (MRCP), endoscopic retrograde cholangiopancreatography (ERCP) and (Computed tomography) CT cholangiography. ERCP is an invasive technique with known complications and hence not preferred in healthy subjects. CT cholangiography may also cause side effects due to the used contrast media, and so is also relatively contraindicated in that group of subjects. MRCP is noninvasive, precise and does not require contrast media making it the most appropriate investigation for preoperative biliary mapping ${ }^{[8]}$.

Detailed preoperative evaluation of the biliary anatomy of the donor in living donor liver transplantation (LDLT) can minimize postoperative morbidity in the recipient and maximize safety for the donor, according to a 2007 study. Conventional magnetic resonance cholangiography was used for depicting the biliary anatomy of LDLT donors. The classical branching pattern of the biliary system was observed in $60.4 \%$ of subjects, with the remaining $39.6 \%$ showing anatomical variations. MRCP showed accurate anatomy of the biliary system having sensitivity in differentiating normal from variant anatomy of $95.5 \%$, specificity of $95.2 \%$, a positive predictive value of 
$96.8 \%$ and a negative predictive value of $93.3 \%{ }^{[9]}$.

A 2003 study stated that surgical procedures such as liver resection and partial liver transplantation are increasing in frequency and complexity, and in hepatic resection for living donor liver transplantation (LDLT), an accurate knowledge of the anatomy of intrahepatic bile ducts (IHDs) is thus critical if the liver is to be successfully harvested and postoperative complications minimized. The anatomy of the intrahepatic bile ducts was typical in $63 \%$ of cases, showed triple confluence in $10 \%$, anomalous drainage of the RPSD into the LHD in 11\%, anomalous drainage of the RPSD into the common hepatic duct (CHD) in 6\%, anomalous drainage of the RPSD into the cystic duct in $2 \%$, drainage of the right hepatic duct (RHD) into the cystic duct, the presence of an accessory duct leading to the CHD or RHD in 5\%, individual drainage of the LHD into the RHD or CHD in $1 \%$, and unclassified or complex variation in $1 \%{ }^{[10]}$.

Another 2008 study tested patients undergoing endoscopic retrograde cholangiograms for different indications. Anatomical variations in IHD were classified according to the branching pattern of the right anterior segmental duct (RASD) and the right posterior segmental duct (RPSD), presence or absence of first-order branch of left hepatic duct (LHD) and of an accessory hepatic duct. Anatomy of the IHD was typical in $52.9 \%$ of cases, showing triple confluence in $11.46 \%$, anomalous drainage of the RPSD into the LHD in $18.2 \%$, anomalous drainage of the RPSD into the common hepatic duct (CHD) in $7.1 \%(\mathrm{n}=18)$, drainage of the right hepatic duct (RHD) into the cystic duct $0.4 \%(\mathrm{n}=$ 1 ), presence of an accessory duct leading to the CHD or RHD in $4.7 \%$, individual drainage of the LHD into the RHD or CHD in $2.4 \%$, and unclassified or complex variations in $2.7 \%^{[11]}$.

\section{Conclusion:}

MRCP is a non-invasive technique that has a high sensitivity in detecting intrahepatic biliary anatomical variants in possible LDLT donors. The most common anatomical variant of biliary tree arborization is the Yoshida RL type where the right anterior and right posterior ducts join to form the right hepatic duct which in turn joins the left intrahepatic duct to form the common hepatic duct. This type is encountered in $74 \%$ of cases. The surgical team must be aware of the donor's anatomical variant preoperatively for a safer, 
more effective procedures and to reduce post-operative complications. MRCP assessment of biliary tree is as essential step in the preoperative evaluation of possible LDLT donors. Detection of intrahepatic biliary anatomical variants helps the surgical team achieve a more effective procedure and reduces post-operative complications

\section{References}

1. Strasberg SM. Nomenclature of hepatic anatomy and resections: a review of the Brisbane 2000 system. J Hepatobiliary Pancreat Surg. 2005;12(5):351-5.

2. Murakami G, Hata F. Human liver caudate lobe and liver segment. Anat Sci Int. 2002;77(4):21124.

3. Clavien, P., \& Baillie, J. (2006). Diseases of the gallbladder and bile ducts: Diagnosis and treatment. Malden, MA: Blackwell Pub.

4. Babu, Ramesh \& Sharma, Malay. (2013). Biliary Tract Anatomy and its Relationship with Venous Drainage. Journal of Clinical and Experimental Hepatology. 4. 10.1016/j.jceh.2013.05.002.

5. Sureka B, Bansal K, Patidar Y, Arora A. Magnetic resonance cholangiographic evaluation of intrahepatic and extrahepatic bile duct variations. Indian J Radiol Imaging. 2016;26(1):22-32.

6. Mortelé KJ, Rocha TC, Streeter JL, Taylor AJ. Multimodality imaging of pancreatic and biliary congenital anomalies. Radiographics. 2006;26(3):715-31.

7. Yoshida J, Chijiiwa K, Yamaguchi K, Yokohata K, Tanaka M. Practical classification of the branching types of the biliary tree: an analysis of 1,094 consecutive direct cholangiograms. J Am Coll Surg. 1996;182(1):37-40.

8. Barsoum NR, Samie AA, Adel A, Asaad RE. Role of MRCP in assessment of biliary variants in living donor liver transplantation. The Egyptian Journal of Radiology and Nuclear Medicine. 2013;44:131-36.

9. Song GW, Lee SG, Hwang S, et al. Preoperative evaluation of biliary anatomy of donor in living donor liver transplantation by conventional nonenhanced magnetic resonance cholangiography. Transpl Int. 2007;20(2):167-73.

10. Choi JW, Kim TK, Kim KW, et al. Anatomic variation in intrahepatic bile ducts: an analysis of intraoperative cholangiograms in 300 consecutive donors for living donor liver transplantation. Korean J Radiol. 2003;4(2):85-90.

11. Sharma V, Saraswat VA, Baijal SS, Choudhuri G. Anatomic variations in intrahepatic bile ducts in a north Indian population. J Gastroenterol Hepatol. 2008;23(7 Pt 2):e58-62.

To cite this article: Medhat M. Reffat, Ahmed E. Shalan, Shehab El-Din Shibl. Role of MRCP in Assessment of Biliary Anatomy in Potential Living Liver Transplant Donors. BMFJ 2021; 38(1): 247253. DOI: $10.21608 / \mathrm{bmfj} .2021 .36939 .1296$ 